\title{
Modelos de estimativa do grau de saturação do concreto a partir das variáveis ambientais aplicados à análise de confiabilidade de estruturas de concreto armado atacadas por íons cloreto
}

\section{Models for estimation of saturation degree of concrete through the environmental variables applied to the reliability analysis of reinforced concrete structures attacked by chloride ions}

\author{
Felipe Favretto ${ }^{1}$, Fábio Costa Magalhães ${ }^{1}$, \\ André Tavares da Cunha Guimarães ${ }^{1}$, Miguel Ángel Climent ${ }^{2}$, \\ Mauro de Vasconcellos Real ${ }^{1}$
}

\footnotetext{
${ }^{1}$ Universidade Federal do Rio Grande, Programa de Pós-graduação em Engenharia Oceânica, Avenida Itália km 8, CEP: 96203-900, Rio Grande-RS, Brasil.

${ }^{2}$ Universidade de Alicante, Departamento de Engenharia Civil, Carretera San Vicente del Raspeig s/n, Ap. 99, CEP: 03080, San Vicente del Raspeig, Alicante, Espanha.

e-mail: f2favretto@gmail.com, fabiocmagalhaes@gmail.com, atcg@vetorial.net,ma.climent@ua.es, mvrealgm@gmail.com
}

\begin{abstract}
RESUMO
A problemática da durabilidade de estruturas de concreto armado inseridas em ambientes de severa agressividade se apresenta como uma das principais dificuldades enfrentadas pela cadeia da construção civil. Dos vários agentes capazes de degradar estas estruturas tem-se atenção especial aos íons cloreto capazes de causar a corrosão de armaduras, estes íons estão amplamente presentes em ambientes marinhos. Neste espectro, a estimativa de vida útil das estruturas de concreto armado se torna uma temática fundamental na avaliação da durabilidade de estruturas. Apesar de vários processos causarem penetração de cloretos no concreto tem-se praticamente como consenso que o mecanismo de difusão é o predominante, tendo-se como um modelo clássico de caracterização da penetração de cloretos a $2^{a}$ Lei da difusão de Fick. O processo de difusão iônica sofre forte influência do grau de saturação (GS) dos poros do concreto, está influencia incide diretamente sobre o coeficiente de difusão de cloretos (D). Ainda que se tenham desenvolvido metodologias de medição do GS elas se apresentam dispendiosas em tempo e recursos. Objetiva-se então apresentar estimativas do Grau de Saturação, baseadas nas condições ambientais de exposição da estrutura, e analisar a influência deste parâmetro na estimativa de vida útil de elementos estruturais de concreto armado sob a ótica da confiabilidade. São utilizados dados meteorológicos e de medição do GS de duas regiões litorâneas, sendo uma em Rio Grande, no sul do Brasil e outra em Santa Pola, sudeste da Espanha. Obtiveram-se modelos de estimativa do GS do concreto para cada situação pesquisada e também para ambos os ambientes em conjunto. Constatou-se então, através de uma abordagem probabilística, a forte influência do uso destes modelos na estimativa de vida útil de elementos de concreto armado.
\end{abstract}

Palavras-chave: Concreto. Ambiente Marinho. Grau de saturação. Durabilidade. Modelagem.

\section{ABSTRACT}

The problematic of the durability of reinforced concrete structures inserted in environments of severe aggressiveness is presented as one of the main difficulties faced by the civil construction chain. Of the various agents capable of degrading these structures, special attention is paid to chloride ions capable of causing corrosion of reinforcing steel, these ions are widely present in marine environments. In this sense, the estimated useful service life of reinforced concrete structures becomes a fundamental theme in the evaluation of the durability of concrete structures. Although several processes cause penetration of chlorides in the concrete, it is practically agreed that the diffusion mechanism is the predominant one, being Fick's $2^{\text {nd }}$ Law of diffusion a 
classic model for the characterization of chloride penetration into concrete. The ionic diffusion process is strongly influenced by the saturation degree (GS) of the pores in the concrete, it influences directly on the chloride diffusion coefficient (D). Although GS measurement methodologies have been developed, they are costly in time and resources. The objective of this study is to present estimates of GS, based on the environmental exposure conditions of the structure, and to analyze the influence of this parameter on the estimated useful service life of structural elements of reinforced concrete from the point of view of reliability. Meteorological and GS measurement data from two coastal regions are used, one in Rio Grande, in southern Brazil and the other in Santa Pola, southeast of Spain. Concrete GS estimation models were obtained for each studied situation and also for both environments together. It was verified, through a probabilistic approach, the strong influence of the use of these models in the estimation of useful service life of reinforced concrete structures.

Keywords: Concrete. Marine Environments. Saturation Degree. Durability. Modeling.

\section{INTRODUÇÃO}

Após o grande advento do concreto armado surgiram diversos casos de deterioração precoce deste tipo de estrutura, o que levou a diversos estudos a respeito do tema. Através de uma necessidade de aperfeiçoar as técnicas e materiais utilizados, visando uma maior competitividade e sustentabilidade do setor, surgiu a necessidade de se abordar os aspectos da vida útil das estruturas ainda na fase de projeto. Entre os diversos problemas enfrentados de deterioração destas estruturas destacam-se os relativos a corrosão de armaduras oriunda do ataque de íons cloreto que penetram na massa de concreto [1-8]. Os íons cloreto estão amplamente difundidos em zonas marítimas, onde se enquadram os ambientes de estudo deste trabalho.

A durabilidade das estruturas de concreto armado pode ser compreendida como o período de tempo no qual a estrutura se comporta de maneira satisfatória, resistindo a todas as solicitações impostas sem apresentar falhas técnicas ou perda da funcionalidade a que se destina. Este período é previamente determinado para o ambiente e as condições inicialmente estimadas $[9,10]$. A durabilidade não é uma propriedade ligada apenas as características da estrutura de concreto, ela resulta dos processos de interação da estrutura com o ambiente em que se encontra inserida. Tendo-se em vista a grande variabilidade dos parâmetros envolvidos nos processos de deterioração há um grande desafio na escolha de modelos determinísticos, probabilísticos ou mistos para estimativa de vida útil das estruturas [7].

A patologia da corrosão das armaduras de concreto é um fator determinante na vida útil das estruturas, porém seus efeitos só são percebidos após um avançado estágio de degradação. Apesar de alguns autores considerarem o período de corrosão das armaduras, denominado propagação, como parte da vida útil da estrutura, outros consideram a despassivação da armadura como o marco que pode delimitar o fim da vida útil. Desta maneira se torna fundamental estimar com maior precisão o tempo em que ocorre a despassivação da armadura, permitindo-se assim tomarem-se medidas de prevenção e manutenção que possam prolongar a vida útil das estruturas [11]. Neste trabalho consideraram-se apenas os efeitos do tempo de despassivação da armadura, ou seja, o período de iniciação da corrosão.

Um modelo clássico do processo de corrosão nas estruturas de concreto armado é o de TUUTTI [12] que divide este processo em duas etapas, sendo a iniciação e a propagação. A iniciação corresponde ao período no qual os íons cloreto se deslocam no interior da massa de concreto, desde a superfície da estrutura até o fim da camada de cobrimento, caracterizando-se assim o atingimento do Estado Limite de Serviço (ELS). A propagação trata dos efeitos da corrosão propriamente dita, ela se inicia na despassivação da armadura e culmina com perda de seção do aço e fissuração do concreto levando a estrutura ao Estado Limite Último (ELU). O período de tempo do estágio de iniciação é consideravelmente maior que o de propagação, logo, os estudos de durabilidade geralmente são focados no período de iniciação [13].

Diversos modelos de representação do processo de iniciação do ataque por cloretos são fundamentados por modelos determinísticos, em geral, a solução da $2^{\mathrm{a}}$ Lei da difusão de Fick. Já é conhecido que a maioria das variáveis envolvidas no processo de iniciação não tem um comportamento determinístico. Em busca de uma solução mais aproximada utiliza-se então uma abordagem probabilística alimentando-se os modelos determinísticos com a consideração da variabilidade das variáveis envolvidas no processo.

Um dos parâmetros mais importantes que governam a solução da $2^{\mathrm{a}}$ Lei de Fick para penetração de cloretos é o coeficiente de difusão de cloretos (D), o processo de difusão dos íons cloreto ocorre apenas em meio aquoso, logo se faz necessária a presença de água na microestrutura de poros do concreto para que haja movimentação iônica. A forte influência do grau de saturação do concreto sobre D já foi comprovada, sendo essencial para correta avaliação da penetração de íons cloreto no concreto [14-19] e [20]. Apesar da comprovada influência do GS sobre D, a medição desta propriedade do concreto enfrenta dificuldades técnicas e 
econômicas, dificultando seu uso. Busca-se então facilitar o processo de obtenção do GS avaliando-se as condições ambientais a que o concreto está exposto.

Diversos fatores podem gerar variação no GS do concreto tais como zona de inserção; características do concreto; posição da superfície exposta em relação a superfície de concretagem; condições de exposição no ambiente tais como sentido da superfície (horizontal ou vertical) e orientação (norte, sul, leste, oeste, para cima e para baixo). Para as diversas situações de exposição relatadas o GS do concreto pode ser influenciado pelas condições meteorológicas locais. PERAÇA [21] relatou que para as condições de exposição de seus testemunhos em Rio Grande no extremo sul do Brasil, as variáveis ambientais mais influentes foram a temperatura máxima (TM) e a umidade relativa (UR). VÍLCHEZ [22] em Santa Pola, Alicante, na Espanha encontrou como variáveis mais influentes a temperatura máxima, taxa de precipitação (TP), horas de insolação (HI) e umidade relativa (UR).

Tendo-se como base dados meteorológicos e de medições de GS realizadas em dois ambientes distintos em zona de névoa marinha, busca-se gerar modelos de GS que permitam estimar com precisão razoável esta propriedade utilizando como parâmetro de entrada as variáveis ambientais. Estas estimativas poderão ser utilizadas na solução dos modelos do período de iniciação para concretos de mesmas características dos pesquisados.

A probabilidade de falha de uma estrutura caracteriza-se como a mensuração probabilística do nível de segurança. Perante a aleatoriedade dos parâmetros envolvidos nos processos geralmente os modelos determinísticos geram imprecisão na estimativa do processo de ataque por cloretos. O uso de modelos probabilísticos se faz fundamental para descrever os processos de deterioração das estruturas de concreto armado. Busca-se utilizar a teoria da confiabilidade na avaliação da influência do GS na estimativa de vida útil, utilizando a despassivação da armadura como o estágio de atingimento do ELS. O objetivo deste trabalho não é aprofundar-se em fundamentos teóricos de confiabilidade, bem como os métodos de solução e aplicação dos procedimentos estocásticos. Mais detalhes sobre estes podem ser obtidos em HALDAR, e MAHADEVAN [23], ANDRADE [24], MAGALHÃES [25] e MELCHERS e BECK [26].

Através das teorias da confiabilidade é possível quantificar as incertezas e determinar a probabilidade de uma estrutura atingir determinado estado limite. Os principais conceitos da confiabilidade estão relacionados ao nível de confiança de que determinada estrutura se comporte satisfatoriamente em serviço. Busca-se para tal, ainda em projeto, especificar uma probabilidade pré-ajustada de falha ou como é esperado de sucesso $[27,28]$

A confiabilidade do problema para duas variáveis pode ser analisada comparando-se capacidade resistente $(\mathrm{R})$ do sistema estrutural com a solicitação $(\mathrm{S})$ a ele imposta, define-se então a função margem de segurança (M) do sistema conforme a Eq. (1).

$M=R-S$

É possível obter-se então o índice de confiabilidade ( $\beta$ ) que é definido pela Eq. (2):

$$
\beta=\frac{\mu_{M}}{\sigma_{M}}
$$

Onde:

$\mu_{M}$ é a média da margem de segurança;

$\sigma_{M}$ é o desvio padrão da margem de segurança;

Através da Figura 1 podemos observar o exposto na Equação 2. 


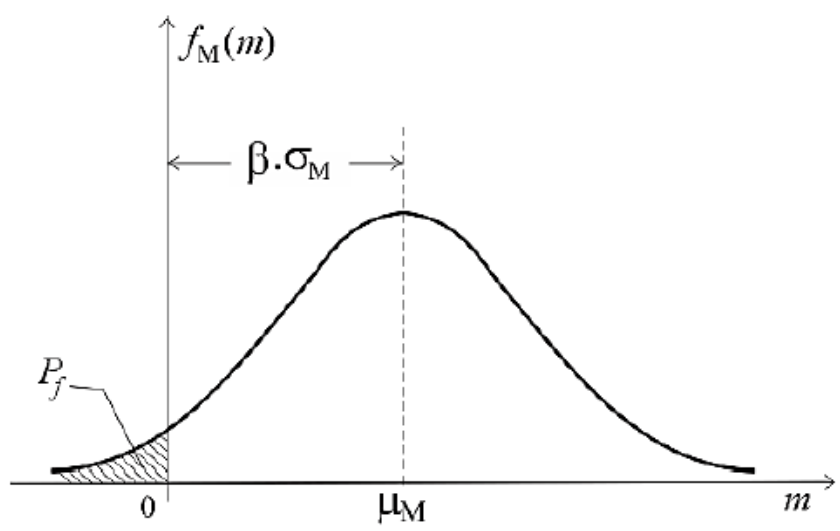

Figura 1: Função de densidade de probabilidades da margem de segurança (Fonte: Adaptado de [29]).

Baseado na geração de números aleatórios com distribuição de probabilidade conhecida para variáveis o método de Simulação de Monte Carlo (MCS) permite realizar uma análise probabilística de determinado fenômeno descrito através da função de falha $g(X)=0$. A realização de um número massivo de simulações permite formar um conjunto de resultados individuais dos quais é possível se obter, através de análise estatística, as características da distribuição de probabilidade das variáveis de resposta do fenômeno estudado [28].

Após iniciado o processo de corrosão das armaduras do concreto sua recuperação se torna extremamente complicada. Devido a esta dificuldade diversos autores [13, 24, 30, 31] adotam o fim do período de iniciação do ataque por cloretos como o atingimento do ELS da estrutura. Segundo MAGALHÃES [25] o processo de deterioração das estruturas atacadas por cloretos se inicia quando o chamado teor crítico ( $\left.C_{\text {Crit }}\right)$ é atingido no ponto estudado. Expressando o estado limite de serviço do elemento estrutural em termos da confiabilidade no que tange a entrada de agentes agressivos obtêm-se a equação 3 . Nesta equação $g(x, t)$ é a função de falha do problema, na qual se compara a concentração de cloretos na armadura considerando-se um cobrimento $x$ e um tempo $t(C(x, t))$ com o teor crítico de cloretos adotado $\left(C_{\text {Crit }}\right)$.

$$
g(x, t)=C_{\text {Crit }}-C(x, t)
$$

A consideração do grau de saturação do concreto na estimativa de vida útil das estruturas é um fator fundamental para se obter maior precisão. A influência deste parâmetro na penetração de íons cloreto no concreto é reconhecidamente um fator determinante. Devido ao fato da mensuração de GS em campo ser uma prática lenta e onerosa, tornando-a pouco recorrente nos estudos de durabilidade, buscou-se a criação de modelos matemáticos que permitem a estimativa do GS do concreto através das variáveis ambientais as quais o elemento estudado está exposto, viabilizando-se assim o uso desta importante propriedade do concreto nos cálculos de vida útil das estruturas de concreto.

\section{MATERIAIS E MÉTODOS}

Para estudar o comportamento do GS em relação às variáveis ambientais, utilizaram-se dados experimentais de medição do GS do concreto e de variáveis meteorológicas de dois macros ambientes distintos, ambos em zona de névoa, um localizado no extremo sul do Brasil na cidade de Rio Grande, Rio grande do Sul, onde mais informações podem ser obtidas em [21] e outro em Santa Pola, Alicante, localizada no sudeste da Espanha onde características mais especificas podem ser obtidas em [22].

Foram utilizados na pesquisa 30 corpos de prova (CPs) de diferentes traços de concreto executados com cimento do tipo CPIV-32 (normatizado pela ABNT NBR 16697). A caracterização dos traços de concreto utilizados está apresentada na tabela 1 . Dos blocos de concreto moldados foram extraídos cilindros de $10 \mathrm{~cm}$ de diâmetro tanto na posição vertical como horizontal e estes fatiados em testemunhos de aproximadamente $4 \mathrm{~cm}$ de altura conforme figura 2. Estes CPs foram expostos primeiramente em uma estação de medição na cidade de Rio Grande, organizados, de acordo com a tabela 2, de forma a contemplar as combinações de traços executados, direções de extração dos cilindros de concreto e testemunhos fatiados (superfície exposta em relação a superfície de concretagem), juntamente com as diferentes possibilidades de exposição das faces (Norte, Sul, Leste, Oeste, para cima e para baixo), possibilitando simular 15 distintas situações, sendo 14 inseridas em ambiente externo e 1 em ambiente interno (Lab). Dos 30 testemunhos expostos na estação de Rio Grande 4 foram levados para a estação de exposição de Santa Pola, permitindo-se obter medi- 
ções de GS destes testemunhos em dois macro ambientes distintos [33].

Tabela 1: Traços de concreto.

\begin{tabular}{l|l|l|l|l|l}
\hline TRAÇO & CIMENTO & AREIA & BRITA & AGUA/CIMENTO & $\begin{array}{l}\text { ABATIMENTO } \\
(\mathrm{mm})\end{array}$ \\
\hline 1 & 1 & 2,12 & 2,88 & 0,54 & 110 \\
\hline 2 & 1 & 1,60 & 2,40 & 0,45 & 110 \\
\hline 3 & 1 & 2,64 & 3,36 & 0,63 & 110 \\
\hline 4 & 1 & 1,60 & 2,40 & 0,54 & 220 \\
\hline 5 & 1 & 2,64 & 3,36 & 0,54 & 120 \\
\hline
\end{tabular}

\begin{tabular}{|c|c|c|}
\hline DIREÇÃO DA EXTRAÇÃO & TESTEMUNHOS FATIADOS & \\
\hline Vertical (V) & Centro $(\mathrm{C})$ & Fundo $(\mathrm{F})$ \\
\hline Horizonta $(\mathrm{H})$ & Centro $(\mathrm{C})$ & \\
\hline
\end{tabular}

Figura 2: Extração e fatiamento dos testemunhos

Tabela 2: Identificação dos testemunhos em Rio Grande-RS e Santa Pola, Alicante.

\begin{tabular}{|c|c|c|c|c|c|c|}
\hline \multirow{2}{*}{ LOCAL } & \multirow{2}{*}{ TESTEMUNHO } & \multirow{2}{*}{ TRAÇO } & \multirow{2}{*}{$\begin{array}{l}\text { DIREÇÃO DE } \\
\text { EXTRAÇÃO * }\end{array}$} & \multirow{2}{*}{$\begin{array}{l}\text { FACE } \\
\text { EXPOSTA* }\end{array}$} & \multicolumn{2}{|c|}{ POSIÇÃO DA FACE EXPOSTA } \\
\hline & & & & & SENTIDO & DIREÇÃO \\
\hline \multirow{15}{*}{$\begin{array}{l}\text { Rio Grande, } \\
\text { RS }\end{array}$} & $1 \mathrm{e} 2$ & 1 & V & $\mathrm{C}$ & V & S \\
\hline & 3 e 4 & 2 & V & $\mathrm{C}$ & V & $S$ \\
\hline & 5 e 6 & 3 & V & $\mathrm{C}$ & V & $S$ \\
\hline & 7 e 8 & 4 & V & $\mathrm{C}$ & V & $S$ \\
\hline & 9 e 10 & 5 & V & $\mathrm{C}$ & V & S \\
\hline & 11 e 12 & 1 & $\mathrm{~V}$ & $\mathrm{~T}$ & $\mathrm{~V}$ & $S$ \\
\hline & 13 e 14 & 1 & V & $\mathrm{F}$ & V & $S$ \\
\hline & 15 e 16 & 1 & $\mathrm{H}$ & $\mathrm{L}$ & V & $S$ \\
\hline & 17 e 18 & 1 & $\mathrm{H}$ & $\mathrm{C}$ & V & $S$ \\
\hline & 19 e 20 & 1 & V & $\mathrm{C}$ & $\mathrm{H}$ & $\mathrm{p} /$ cima \\
\hline & 21 e 22 & 1 & V & $\mathrm{C}$ & V & $\mathrm{L}$ \\
\hline & 23 e 24 & 1 & V & $\mathrm{C}$ & $\mathrm{H}$ & $\mathrm{p} /$ baixo \\
\hline & 25 e 26 & 1 & V & $\mathrm{C}$ & V & $\mathrm{N}$ \\
\hline & 27 e 28 & 1 & V & $\mathrm{C}$ & V & $\mathrm{O}$ \\
\hline & 29 e 30 & 1 & V & $\mathrm{C}$ & V & Lab \\
\hline \multirow{4}{*}{$\begin{array}{l}\text { Santa Pola, } \\
\text { Alicante }\end{array}$} & 30 & 1 & V & $\mathrm{C}$ & V & $\mathrm{N}$ \\
\hline & 31 & 2 & V & $\mathrm{C}$ & V & $\mathrm{N}$ \\
\hline & 35 & 4 & V & C & V & $\mathrm{N}$ \\
\hline & 36 & 4 & V & $\mathrm{C}$ & V & $\mathrm{N}$ \\
\hline
\end{tabular}

\footnotetext{
*em relação a superfície de concretagem
} 
Os dados meteorológicos pertinentes a estação de medição de GS de Rio Grande foram obtidos junto ao Instituto de Oceanografia da Universidade Federal do Rio Grande e foram os seguintes: pressão atmosférica (PA), temperatura de ar seco (TAS), temperatura máxima (TM), temperatura mínima (TMin), umidade relativa (UR), taxa de precipitação (TP), taxa de evaporação (TE), temperatura de ar úmido (TAU), horas de insolação (HI), nebulosidade (N), visibilidade (V) e taxa de evaporação externa (TEE). Já os dados meteorológicos relacionados a estação de medição de GS de Santa Pola foram obtidos da rede de dados abertos da agência estatal de meteorologia (AEMET) espanhola, a estação meteorológica de referência para a obtenção dos dados foi a instalada no aeroporto de El Altet, localizado no distrito de El Altet na cidade de Elche, os dados obtidos foram temperatura máxima, temperatura mínima, temperatura média (TMed), taxa de precipitação, horas de insolação, pressão atmosférica máxima (PAMax), pressão atmosférica mínima (PAMin) e consequentemente a pressão atmosférica média (PA). Não foi possível obter-se dados de UR da referida estação meteorológica desta forma impossibilitando a análise de sua influência no GS dos testemunhos expostos em Santa Pola e também sua comparação com os dados dos testemunhos de Rio Grande.

Visando-se avaliar a influência de cada variável meteorológica sobre o GS do concreto fez-se uso da correlação para avaliar-se a intensidade de interação destas com o GS de cada família de concreto, permitindo-se assim elencar as melhores variáveis para a construção dos modelos de GS. A correlação estatística representa o quão fortemente as variáveis se relacionam em uma amostragem. Através do parâmetro de correlação (r) permite-se fazer inferências sobre o comportamento de uma variável em relação a outra para um espaço amostral definido, como por exemplo, a possível influência de uma variável sobre a outra, a intensidade e o sentido da interação entre estas [33].

A obtenção de modelos de geração de GS alimentados pelas variáveis meteorológicas requer a análise de um conjunto de dados consistente que represente todas as variáveis do processo. A regressão linear baseiase na realização de uma análise estatística para a obtenção de um modelo matemático que permite a relação entre uma variável dependente $(\mathrm{Y})$ com uma variável (X) (regressão linear simples) ou mais variáveis independentes $\left(\mathrm{x}_{1}, \mathrm{x}_{2}, \ldots \mathrm{x}_{\mathrm{n}}\right)$ (regressão linear múltipla). Deste modo, a regressão consiste na geração de uma equação que explica a variação da variável dependente em função de uma ou mais das variáveis independentes. $\mathrm{O}$ parâmetro que infere a precisão do modelo obtido é chamado de coeficiente de determinação $\left(\mathrm{r}^{2}\right)$, que varia de 0 a 1 e expressa quanto por cento dos resultados da variável dependente podem ser obtidos no modelo através da (s) variável (s) independente (s). A representação gráfica deste fenômeno pode ser feita através do diagrama de dispersão, para verificar como se comportam os valores da variável dependente $\mathrm{Y}$ em função da variação da variável independente X [34].

De posse das variáveis ambientais de melhor correlação com o GS realizou-se regressões lineares múltiplas para todos os arranjos propostos, permitindo-se assim avaliar o coeficiente de determinação $\mathrm{r}^{2} \mathrm{de}$ cada modelo. Foram gerados modelos através da regressão linear para todas as famílias de concreto expostas, também foram gerados modelos para as famílias de concreto expostas tanto no Brasil quanto na Espanha através de todos os dados agrupados. Para avaliar um possível comportamento mais geral do GS avaliou-se também modelos gerados para as médias do GS de algumas famílias de concreto.

Os processos matemáticos envolvidos na geração de modelos de estimativa através da regressão linear geram imprecisão nos resultados obtidos, seja por fatores inerentes as medições das variáveis ou pelo simples fato da aleatoriedade de algumas das variáveis envolvidas no processo. Buscando-se avaliar a qualidade dos modelos obtidos e realizar algumas comparações entre eles calculou-se o erro médio percentual absoluto de cada modelo, comparando-se através da equação 4 o valor obtido do modelo matemático com o valor real do parâmetro.

$$
\operatorname{ERRO}(\%)=\frac{Y_{E S T .}-Y_{R E A L}}{Y_{R E A L}} \times 100
$$

Para a aplicação das teorias da confiabilidade elencou-se como modelo de simulação da entrada de agentes agressivos no concreto a $2^{\mathrm{a}}$ Lei da difusão de Fick. Adotou-se como critério de falha o atingimento de um teor crítico de cloretos na profundidade de cobrimento, correspondendo ao atingimento do ELS do elemento estrutural analisado. Para considerar a influência de alguns fatores que afetam a penetração de cloretos no concreto adotou-se os coeficientes de correção do coeficiente de difusão de referência do concreto 
$\mathrm{D}_{0}$ propostos por GUIMARÃES [35]. O modelo final adotado para a representação da concentração de cloretos pela profundidade a determinado tempo é expresso pela equação 5.

$$
C_{(x, t)}=C_{S^{*}} \operatorname{erfc}\left(\frac{x}{2 \sqrt{R_{C} \cdot R_{G S} \cdot R_{T} \cdot R_{S C} \cdot D_{0} \cdot t}}\right)
$$

Onde:

$C_{S}$ é a concentração superficial de cloretos em função do tempo; erfc( ) é a função complementar erro de Gauss;

$\mathrm{R}_{\mathrm{C}}$ é o coeficiente de correção devido à influência do cimento utilizado;

$\mathrm{R}_{\mathrm{GS}}$ é o coeficiente de correção devido à influência do GS do concreto;

$\mathrm{R}_{\mathrm{T}}$ é o coeficiente de correção devido à influência da temperatura ambiente;

$\mathrm{R}_{\mathrm{SC}}$ é o coeficiente de correção devido à influência da superfície exposta em relação à concretagem;

$\mathrm{D}_{0}$ é o coeficiente de difusão de referência do concreto.

Visando-se efetuar a verificação do processo de simulação utilizaram-se dados experimentais relativos a elementos estruturais de concreto armado, inseridos em ambiente de severa agressividade, apresentados por GUIMARÃES [35]. Estes resultados foram obtidos de estruturas do cais Terminal de Conteiners (TECON) do Superporto da cidade de Rio Grande - RS [36] e [37]. Utilizaram-se os dados relativos ao elemento denominado de PS pelos autores, devido a estar inserido em zona totalmente de névoa, condizendo com o ambiente de exposição dos testemunhos desta pesquisa.

Para a consideração probabilística da equação 4 foram utilizados os valores médios e os respectivos coeficientes de variação de cada parâmetro na geração de números aleatórios. A influência do GS na penetração de cloretos é considerada através do parâmetro de correção $\mathrm{R}_{\mathrm{GS}}$, sendo assim, a única variável da equação que não foi obtida diretamente da bibliografia foi o $\mathrm{R}_{\mathrm{GS}}$ gerado através dos modelos de GS, para facilitar o entendimento do problema resumiu-se as variáveis de entrada do modelo na tabela 3.

Tabela 3: Parâmetros e distribuição de probabilidades das variáveis utilizadas na avaliação da confiabilidade.

\begin{tabular}{|c|c|c|c|c|c|}
\hline VARIÁVEL & UNIDADE & MÉDIA & $\begin{array}{c}\text { COEF. DE } \\
\text { VARIAÇÃO }\end{array}$ & $\begin{array}{c}\text { TIPO DE } \\
\text { DISTRIBUIÇÃO }\end{array}$ & REFERÊNCIA \\
\hline$C_{S}$ & - & 2,46 & 0,20 & $\mathrm{LN}$ & YU et al. [13] \\
\hline \multirow{2}{*}{$C_{\text {crit }}$} & \multirow{2}{*}{$\%$} & \multirow{2}{*}{0,40} & \multirow{2}{*}{0,15} & \multirow{2}{*}{$\mathrm{N}$} & HELENE [7] \\
\hline & & & & & YU et al. [13] \\
\hline \multirow{3}{*}{$\mathrm{R}_{\mathrm{GS}}$} & - & 0,205 & 0,05 & $\mathrm{LN}$ & GUIMARÃES [37]* \\
\hline & - & 1,00 & 0,00 & - & - \\
\hline & - & \multicolumn{3}{|c|}{ Conforme modelos de GS } & Autor \\
\hline $\mathrm{R}_{\mathrm{T}}$ & - & 0,81 & 0,20 & $\mathrm{~N}$ & BRETANHA [36]* \\
\hline $\mathrm{R}_{\mathrm{SC}}$ & - & 0,74 & 0,20 & $\mathrm{LN}$ & GUIMARÃES [35]* \\
\hline $\mathrm{R}_{\mathrm{C}}$ & - & 0,64 & 0,00 & - & - \\
\hline $\mathrm{D}_{0}$ & $\mathrm{~cm}^{2} / \mathrm{ano}$ & 104 & 0,10 & $\mathrm{~N}$ & BENTZ [30] \\
\hline$x$ & $\mathrm{~cm}$ & $x_{\text {MÉDIO }}$ & 0,10 & $\mathrm{~N}$ & MAGALHÃES [28] \\
\hline
\end{tabular}

$\mathrm{N}$ - Normal; LN - LogNormal; *obtido através dos dados apresentados pelos autores.

Pelo viés do modelo proposto avaliou-se a confiabilidade através da probabilidade de falha do sistema no atingimento do ELS, calculada utilizando-se a equação 6, realizando-se para tal 1.000.000 de simulações pelo Método de Monte Carlo. Para execução das simulações com a consideração de cada modelo de GS foi programado um código em linguagem Octave para cada caso, tendo-se desenvolvido 8 rotinas para simulação do problema proposto. Desenvolveu-se uma rotina para cada modelo de GS apresentado, uma desconsiderando o coeficiente de correção $\mathrm{R}_{\mathrm{GS}}$, ou seja, considerando o grau de saturação igual a $100 \%$ e também uma para $\mathrm{R}_{\mathrm{GS}}=0,205$ [37] com coeficiente de variação igual a 0,05 . 


$$
P_{f}=\frac{N f}{n}
$$

Onde:

Pf é a probabilidade de falha do sistema.

Nf é o número total de falhas nas simulações realizadas.

n é o número total de simulações realizadas..

\section{RESULTADOS}

Através das correlações realizadas entre todas as variáveis fez-se possível elencar as mais representativas para a geração de cada modelo de GS, obtendo-se as variáveis mais influentes de cada ambiente distinto e também dos dados conjuntos de ambos os ambientes. As variáveis de melhor coeficiente de correlação $r$ são apresentadas nas tabelas 4 e 5 para médias semanais e sazonais das variáveis.

Tabela 4: Resultado das correlações das variáveis com GS para médias semanais.

\begin{tabular}{|c|c|c|c|}
\hline VARIÁVEIS & TAS & TM & TE \\
\hline GS CPS $1^{*}$ e $2^{*}$ & $-0,7087$ & $-0,746$ & $-0,6826$ \\
\hline CPS $1^{*}$ a $28^{*}$ & $-0,6286$ & $-0,6726$ & $-0,6198$ \\
\hline VARIÁVEIS & TM & Tmed & TP \\
\hline GS (30) & $-0,3774$ & $-0,3389$ & 0,518 \\
\hline CPS $(30,31,35$ e 36$)$ & $-0,3994$ & $-0,3593$ & 0,5194 \\
\hline GS CP (30), $1^{*}$ E 2* & $-0,4327$ & $-0,3616$ & 0,4335 \\
\hline $\operatorname{CPS}(30,31,35$ e 36$) E 1^{\star}, 2^{\star}, 3^{\star}, 4^{\star}, 7^{\star}$ e $8^{\star}$ & $-0,318$ & $-0,2426$ & 0,4381 \\
\hline
\end{tabular}

* Testemunho exposto em Rio Grande. ( ) Testemunho exposto em Santa Pola.

Tabela 5: Resultado das correlações das variáveis com GS para médias sazonais.

\begin{tabular}{|c|c|c|c|}
\hline VARIÁVEIS & TAS & TM & Tmín \\
\hline GS CPS $1^{\star}$ e $2^{*}$ & $-0,8646$ & $-0,8776$ & $-0,8495$ \\
\hline VARIÁVEIS & TAS & TM & TAU \\
\hline Média (r) CPS $1^{\star}$ a $28^{\star}$ & $-0,7695$ & $-0,7935$ & $-0,7524$ \\
\hline VARIÁVEIS & TM & Tmed & TP \\
\hline GS (30) & $-0,5471$ & $-0,5352$ & 0,5088 \\
\hline Média (r) CPS $(30,31,35$ e 36) & $-0,5643$ & $-0,5447$ & 0,6376 \\
\hline VARIÁVEIS & TP & HI & PA \\
\hline GS CP (30), $1^{*}$ E 2* & 0,7673 & $-0,4564$ & 0,4624 \\
\hline $\begin{array}{c}\text { Média (r) CPS }(30,31,35 \text { e } 36) E 1^{\star}, 2^{\star}, 3^{\star}, 4^{\star}, 7^{\star} \\
\text { e } 8^{*}\end{array}$ & 0,8257 & $-0,4838$ & 0,5317 \\
\hline
\end{tabular}

* Testemunho exposto em Rio Grande. ( ) Testemunho exposto em Santa Pola.

Comparando-se as tabelas 2 e 3 é possível observar que para praticamente todos os casos as médias sazonais das variáveis geram uma melhor correlação dos dados analisados, consequentemente, uma maior precisão nos modelos por elas gerados. Elencou-se então para a geração dos modelos de estimativa do GS do concreto as variáveis agrupadas em média sazonais, obtendo-se a partir da regressão linear múltipla as equações de GS para cada arranjo estudado bem como o coeficiente de determinação $\mathrm{r}^{2}$, que mede o grau de ajuste do modelo gerado aos dados experimentais. Os resultados são apresentados na tabela 6.

Tabela 6: Modelos de GS obtidos da regressão linear múltipla para médias sazonais das variáveis.

\begin{tabular}{c|c|c}
\hline TESTEMUNHO & EQUAÇÃO & $\mathbf{r}^{\mathbf{2}}$ \\
\hline $\mathbf{1}^{\boldsymbol{*}} \mathbf{e} \mathbf{2}^{\boldsymbol{*}}$ & $87,3333-6,3231 * \mathrm{TAS}+0,6584 * \mathrm{TM}+4,9773 * \mathrm{TMín}$ & 0,7989 \\
\hline $\mathbf{1}^{\boldsymbol{}} \mathbf{a} \mathbf{2 8}^{\boldsymbol{*}}$ & $81,2694+1,3410 * \mathrm{TAU}+0,7468 * \mathrm{TAS}-2,5397 * \mathrm{TM}$ & 0,8241 \\
\hline
\end{tabular}




\begin{tabular}{c|c|c}
\hline TESTEMUNHO & EQUAÇÃO & $\mathbf{r}^{\mathbf{2}}$ \\
\hline $\mathbf{( 3 0 )}$ & $47,9736+1,2377 * \mathrm{TM}-1,4277 * \mathrm{TMéd}+2,8620 * \mathrm{TP}$ & 0,4096 \\
\hline $\mathbf{( 3 0}, \mathbf{3 1}, \mathbf{3 5} \mathbf{e} \mathbf{3 6})$ & $46,9474+0,5708 * \mathrm{TM}-0,7549 * \mathrm{TMéd}+3,1438 * \mathrm{TP}$ & 0,4592 \\
\hline $\mathbf{1}^{\star}, \mathbf{2}^{\star} \mathbf{e} \mathbf{( 3 0 )}$ & $-20,3022+2,0231 * \mathrm{TP}-0,0148 * \mathrm{HI}+0,0713 * \mathrm{PA}$ & 0,5934 \\
\hline $\begin{array}{c}\mathbf{( 3 0}, \mathbf{3 1}, \mathbf{3 5} \mathbf{e} \mathbf{3 6}) \mathbf{e} \\
\mathbf{1}^{*}, \mathbf{2}^{*}, \mathbf{3}^{*}, \mathbf{4}^{\star}, \mathbf{7}^{*}, \mathbf{8}^{*}\end{array}$ & $-201,1000+3,2475 * \mathrm{TP}+0,1628 * \mathrm{HI}+0,2451 * \mathrm{PA}$ & 0,7400 \\
\hline
\end{tabular}

* Testemunho exposto em Rio Grande. ( ) Testemunho exposto em Santa Pola.

O processo de regressão linear consiste no ajuste de uma equação matemática ao conjunto de dados experimentais. Neste processo são inúmeros os fatores que podem ocasionar erros. Para avaliar os erros na estimativa do GS através dos modelos propostos gerou-se para cada modelo o erro absoluto médio percentual, ou seja, considerou-se como erro do modelo a média do valor absoluto dos erros de cada valor estimado pelo modelo quando comparado ao dado experimental medido, os resultados obtidos constam na tabela 7 .

Tabela 7: Erro absoluto médio percentual dos modelos de GS.

\begin{tabular}{c|c}
\hline MODELO GS & Erro médio (\%) \\
\hline $\mathbf{1}^{\star}$ e $\mathbf{2}^{\star}$ & $2,11 \%$ \\
\hline $\mathbf{1}^{\star} \mathbf{a} \mathbf{\mathbf { 8 } ^ { \star }}$ & $1,79 \%$ \\
\hline $\mathbf{( 3 0 )}$ & $2,98 \%$ \\
\hline $\mathbf{( 3 0}, \mathbf{3 1}, \mathbf{3 5}$ e $\mathbf{3 6})$ & $2,57 \%$ \\
\hline $\mathbf{1}^{\star}, \mathbf{2}^{\star}$ e (30) & $4,09 \%$ \\
\hline $\mathbf{( 3 0}, \mathbf{3 1}, \mathbf{3 5}$ e $\mathbf{3 6})$ e $\mathbf{1}^{\star}, \mathbf{2}^{\star}, \mathbf{3}^{\star}, \mathbf{4}^{\star}, \mathbf{7}^{\star}, \mathbf{8}^{\star}$ & $5,47 \%$
\end{tabular}

* Testemunho exposto em Rio Grande. ( ) Testemunho exposto em Santa Pola.

Para a geração de números aleatórios no processo de Simulação de Monte Carlo se faz necessário conhecer o tipo de distribuição de probabilidade de cada variável do modelo. No caso do GS, os parâmetros de entrada são as variáveis meteorológicas. Através da análise estatística e do teste de aderência de Kolmogorov Smirnov analisou-se o tipo de distribuição de probabilidade de cada variável utilizada, bem como os valores da média, desvio padrão e correlação entre variáveis. Um exemplo de histograma e curva de distribuição de probabilidade é apresentado na figura 3, as demais variáveis são apresentadas na tabela 8. Haja vista a correlação existente entre as variáveis de entrada dos modelos de GS se faz necessário utilizar este parâmetro na geração de números aleatórios, evitando-se assim a geração de valores extremos opostos que gerarão estimativas imprecisas do GS, para tal analisou-se a correlação existente entre as variáveis de cada modelo, as quais são apresentadas na tabela 9.

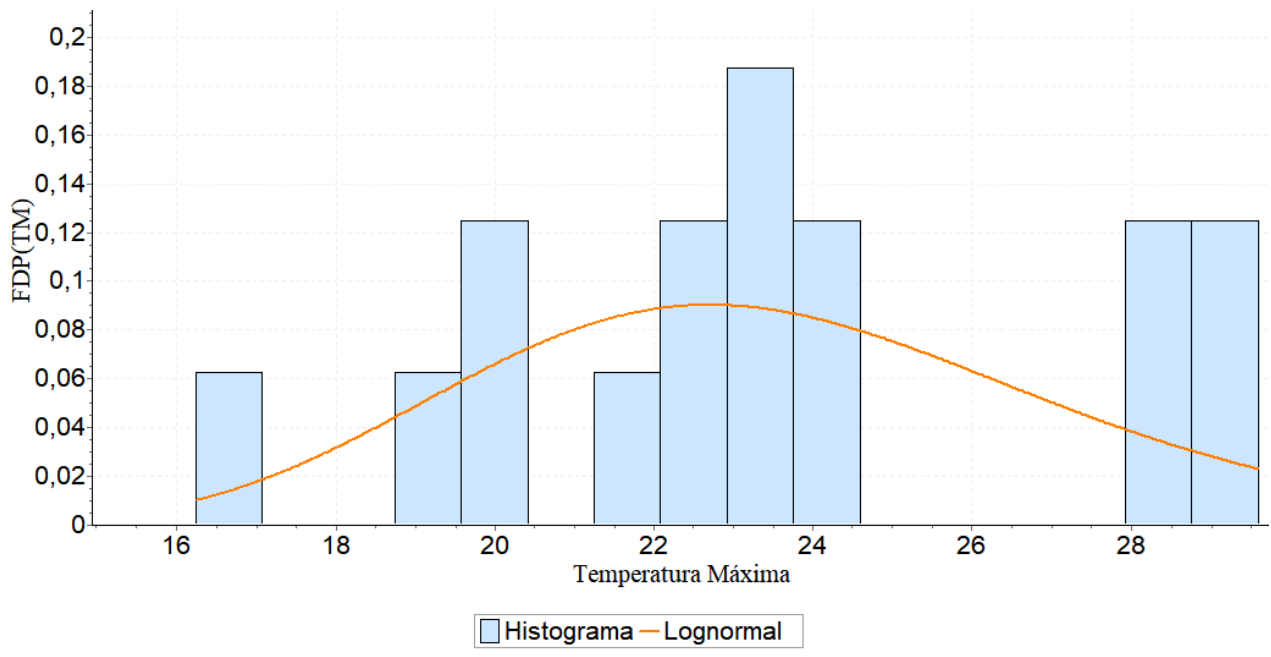

Figura 3: Histograma Função Densidade de Probabilidade da temperatura máxima para média sazonal da estação de Rio Grande 
Tabela 8: Parâmetros estatísticos das variáveis de entrada dos modelos de GS.

\begin{tabular}{c|c|c|c}
\hline VARIÁVEL & DIST. EST. & $\boldsymbol{\mu}$ & $\boldsymbol{\sigma}$ \\
\hline TAS RG & Normal & 18,623 & 3,626 \\
\hline TM RG & Log Normal & 23,589 & 3,82 \\
\hline TMín RG & Normal & 14,909 & 3,482 \\
\hline TAU RG & Normal & 16,954 & 3,135 \\
\hline TM AL & Log Normal & 23,225 & 4,706 \\
\hline TP AL & Weibull & 0,557 & 0,469 \\
\hline TMéd AL & Log Normal & 18,283 & 4,951 \\
\hline TP RG + AL & Weibull & 1,857 & 1,722 \\
\hline HI RG + AL & Uniforme & 7,1623 & 1,577 \\
\hline PA RG + AL & Normal & 1011,9 & 6,073 \\
\hline
\end{tabular}

Tabela 9: Correlação das variáveis de entrada dos modelos de GS.

\begin{tabular}{c|c}
\hline VARIÁVEIS & $\boldsymbol{r}$ \\
\hline TAS e TM RG & 0,9933 \\
\hline TAS e TMín RG & 0,9982 \\
\hline TM e TMín RG & 0,9857 \\
\hline TAU e TAS RG & 0,9892 \\
\hline TAU e TM RG & 0,9892 \\
\hline TM e TP AL & $-0,377$ \\
\hline TM e TMéd AL & 0,9983 \\
\hline TP e TMéd AL & $-0,3396$ \\
\hline TP e HI RG + AL & $-0,4392$ \\
\hline TP e PA RG + AL & 0,4527 \\
\hline HI e PA RG + AL & $-0,762$ \\
\hline
\end{tabular}

Mesmo adotando-se a correlação existente entre as variáveis geradoras de GS este apresenta alguns valores extremos oriundos dos erros acumulados em todas as etapas do processo de estimativa. Visando-se mitigar a influência dos valores extremos de GS na análise de confiabilidade optou-se por truncar os valores de GS obtido em cada modelo respectivamente em 5\% abaixo e acima do menor e do maior valor do GS medido para cada arranjo de dados. Os valores de truncagem de cada modelo são apresentados na tabela 10. 
Tabela 10: Valores de truncagem de GS.

\begin{tabular}{c|c|c}
\hline MODELO & GS Máximo & GS Mínimo \\
\hline $1 * \mathrm{e} 2 *$ & 70,01 & 51,53 \\
\hline$(30)$ & 60,79 & 45,25 \\
\hline $1 *, 2 * \mathrm{e}(30)$ & 70,01 & 45,25 \\
\hline 1 a $28 *$ & 67,7 & 51,24 \\
\hline$(30,31,35$ e 36) & 55,89 & 41,82 \\
\hline $1 *, 2^{*}, 3 *, 4 *, 7 *, 8 * \mathrm{e}(30,31,35,36)$ & 71,92 & 41,88 \\
\hline
\end{tabular}

* Testemunho exposto em Rio Grande. ( ) Testemunho exposto em Santa Pola.

A consideração do GS do concreto na estimativa da concentração de cloretos e consequente determinação da probabilidade de falha não é feita de forma direta. A consideração deste parâmetro é feita através do fator de correção $\mathrm{R}_{\mathrm{GS}}$, que leva em conta a influência do GS do concreto no coeficiente de difusão de cloretos. Para geração do $\mathrm{R}_{\mathrm{GS}}$ em função do GS obtido dos modelos de estimativa utilizou-se dados de GUIMARÃES [37] na geração de uma equação através da regressão linear simples. O modelo obtido na equação 7 se comporta satisfatoriamente para valores de GS entre 40 a $90 \%$, faixa na qual estão compreendidos os valores de truncagem de GS.

$$
\text { Rgs }=-0,2789+0,006528 * G S \quad \text { p/ } \quad 40 \%<G S<90 \%
$$

Para avaliar o número de simulações necessário para a precisão dos resultados avaliou-se a convergência estatística da média e do desvio padrão da concentração de cloretos para um tempo de 50 anos e diversos cobrimentos com todos os modelos de GS utilizados, bem como para os valores de $R_{\mathrm{GS}}=0,205$ e $\mathrm{R}_{\mathrm{GS}}$ $=1$. Os resultados utilizando o modelo $\mathrm{R}_{\mathrm{GS}}=0,205$ são apresentados na figura 4, os demais modelos também apresentaram convergência satisfatória para o número de simulações utilizado. Apesar de haver uma boa convergência para 10.000 simulações optou-se por utilizar 1.000 .000 de iterações no processo.

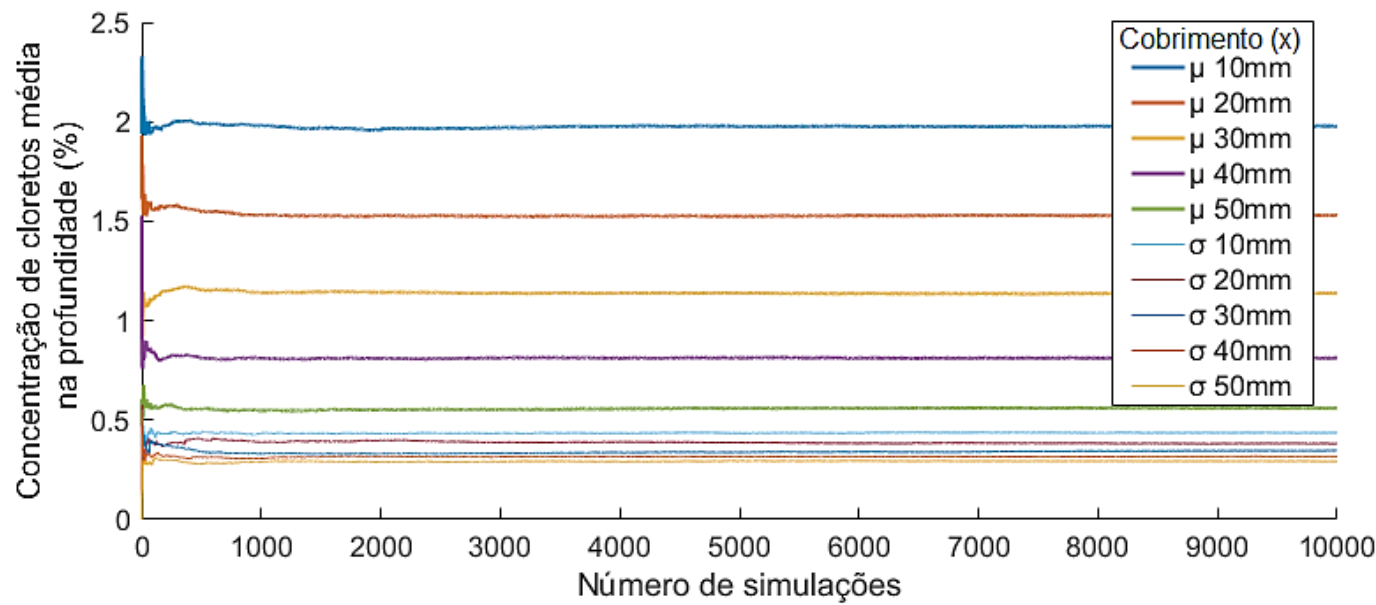

Figura 4: Convergência da média e desvio padrão da concentração de cloretos em 50 anos para diversos valores de cobrimento.

Para possibilitar a comparação do efeito dos modelos de GS propostos em um caso real analisou-se a concentração média de cloretos na profundidade obtida para um tempo de 22 anos, idade na qual os perfis obtidos podem ser comparados ao medido por GUIMARÃES [35], o resultado é apresentado na figura 5. Utilizou-se para esta simulação todos os parâmetros apresentados na tabela 3 bem como os modelos de GS apresentados na tabela 4 e suas respectivas variáveis de entrada. 


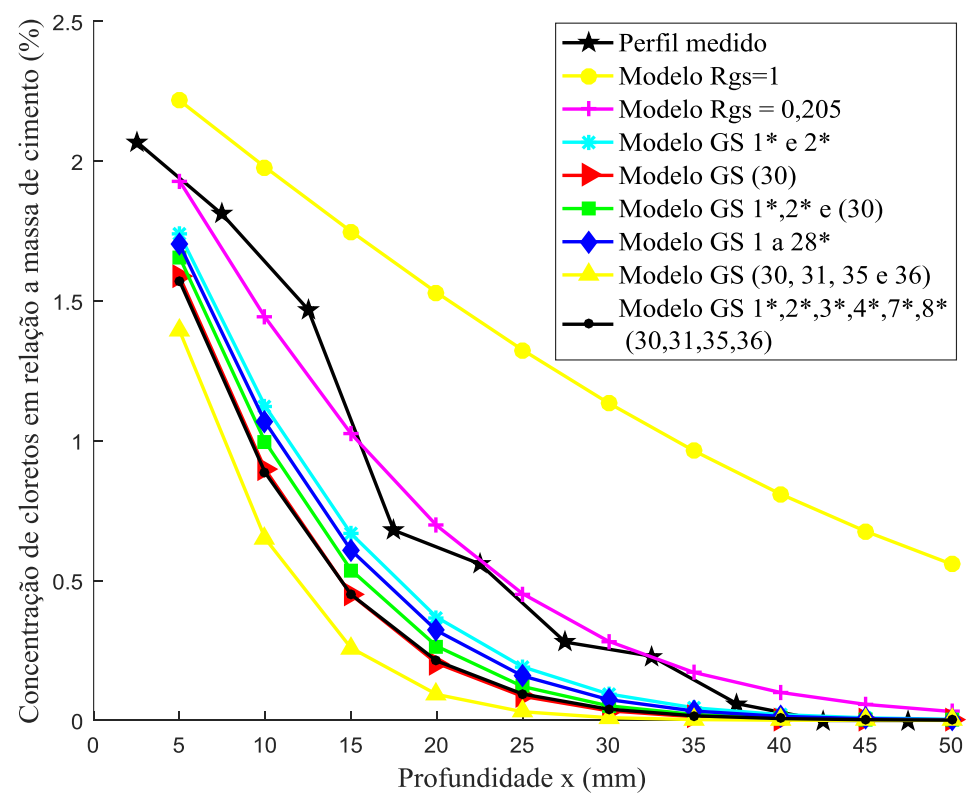

Figura 5: Perfis de penetração de cloretos obtidos da simulação de cada modelo proposto e perfil medido por GUIMARÃES [35].

Através da figura 5 é possível comparar o perfil da concentração de cloretos média gerado por cada modelo com o perfil real de cloretos obtido por GUIMARÃES [35]. Nota-se que o modelo desconsiderando o fator de correção $R_{G S}$ fica bastante distante da curva real, já considerando $R_{G S}=0,205$ há uma melhor aproximação cum o perfil medido. Para os modelos de GS propostos neste trabalho apresentou-se para todos os casos perfis que subestimam o perfil real de cloretos, porém, apresentam comportamento semelhante ao real. O modelo de GS mais ajustado foi o obtido dos CPs 1 e 2 da estação de Rio Grande, que de fato apresenta a maior similaridade com as características da estrutura estudada.

O fato de obter-se um GS menor nos modelos propostos neste trabalho pode ser explicado em parte pela localização das estações de medição em relação à costa além das diferenças climáticas entre as regiões. Considerando o modelo desta pesquisa mais similar a curva real, oriundo do GS dos CPs 1 e 2 de Rio Grande, temos um GS médio de 59,32 a 1200 metros da linha d'água, contra 77,5 obtido por GUIMARÃES [35] a 120 metros da costa e 74,44 obtido por GUIMARÃES [37] junto à costa. Esta situação reforça os estudos de Meira (2004) que constatou uma variação do GS do concreto conforme há afastamento da linha costeira.

Visando-se uma comparação mais adequada dos perfis obtidos nesta pesquisa ao medido por GUIMARÃES [35], admitiu-se a relação do GS com o local de exposição, e, na falta de metodologia mais adequada, efetuou-se uma correção percentual sobre o GS obtido dos modelos deste trabalho para com o obtido por GUIMARÃES [37] na estrutura do cais Terminal de Containers (TECON) do superporto da cidade de Rio Grande - RS. A correção foi efetuada através da diferença percentual do GS obtido por GUIMARÃES [37] junto ao ponto PS para com o testemunho de maior semelhança exposto na estação de medição de Rio Grande, no caso os CPs 1 e 2. O GS médio das estações sazonais dos CPs 1 e 2 da estação de medição de Rio Grande, foi de 59,32 já o obtido por GUIMARÃES [37] foi de 74,44 isto representa um aumento de 25,49\% no GS. Aplicou-se então está diferença percentual sobre o GS obtido de todos os modelos, permitindo-se assim comparar os diferentes modelos de GS junto ao ponto PS, a concentração média de cloretos na profundidade obtida das simulações com GS corrigido para um tempo de 22 anos é apresentada na figura 6. 


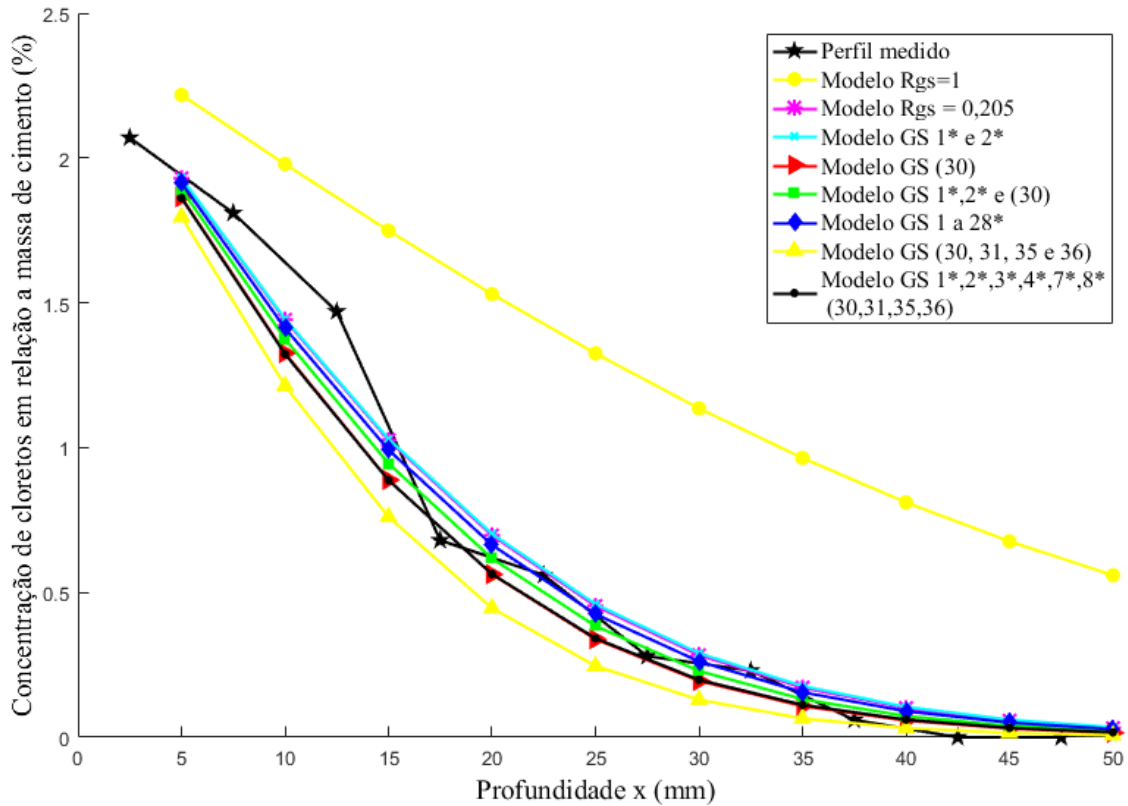

Figura 6: Perfis de penetração de cloretos obtidos da simulação de cada modelo proposto com correção de GS para o ponto PS e perfil medido por GUIMARÃES [35].

Utilizando-se a correção proposta é possível observar um ajuste quase perfeito do modelo de GS $1 *$ e $2^{*}$ ao modelo de $\mathrm{R}_{\mathrm{GS}}$ igual a 0,205 , os quais foram os parâmetros de referência da correção, estando o resultado de acordo com esperado. Sendo assim se faz possível analisar a proximidade dos resultados obtidos em todos os demais modelos, desde que, considerada a correção do GS devido a influência do local de exposição.

Para avaliar o impacto do uso dos diferentes modelos de estimativa do GS sobre a estimativa de vida útil de elementos estruturais de concreto armado, optou-se por avaliar a probabilidade de falha da estrutura no atingimento do ELS em um tempo de 50 anos. A probabilidade de falha de cada modelo foi gerada em função de diversos cobrimentos, os resultados obtidos são apresentados na figura 7.

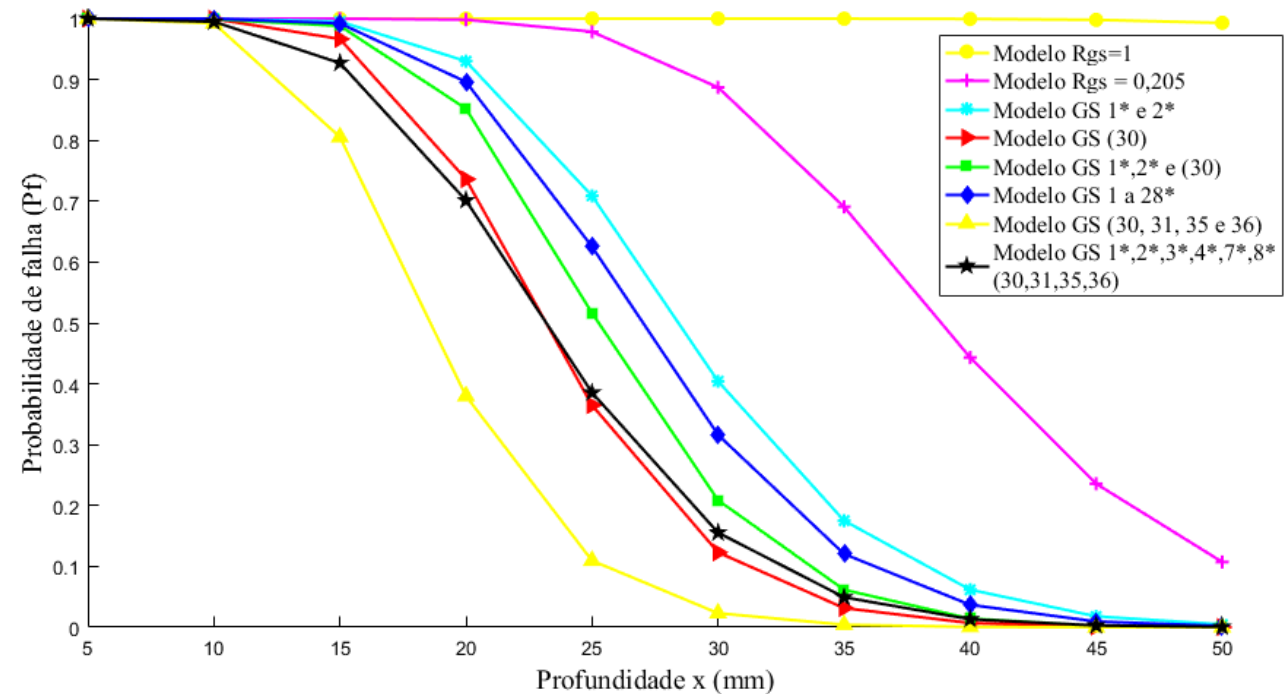

Figura 7: Probabilidade de falha dos modelos utilizados na simulação para diferentes cobrimentos.

Avaliando-se os diferentes modelos e configurações de GS utilizados na estimativa da probabilidade de falha do elemento estrutural analisado pode-se notar a grande discrepância entre eles. Nota-se que para o modelo considerando o concreto saturado em todo o período de tempo há uma elevada probabilidade de falha, provavelmente não retratando a situação real da estrutura. Comparando-se os modelos de GS propostos ao modelo utilizando $\mathrm{R}_{\mathrm{GS}}=0,205$ proposto por GUIMARÃES [37] há uma diferença considerável, indicando que o GS obtido dos modelos é, para todos os casos, inferior ao medido pelo referido autor, tal fato pode ser 
explicado possivelmente pela distância das estações de medição de GS em relação a linha d'água, situação que segundo MEIRA [38] influi no GS do concreto. Esta diferença entre o GS medido na estação de medição de Rio Grande e o medido junto ao cais do TECON também foi constatada por RODRIGUES [39], que analisou a variação do GS de testemunhos executados com cimento de alta resistência inicial, embora o cimento utilizado seja diferente há uma grande similaridade nos resultados encontrados, evidenciando a influência da relação do GS com a distância da costa.

Comparando-se os modelos de GS propostos pode-se notar que há diferenças consideráveis entre eles, principalmente, no que se refere ao ambiente de exposição que o concreto se encontra. Apesar de todos os estudos citados serem realizados em microambiente de névoa as diferenças do local de exposição e características ambientais podem levar a um diferente GS até mesmo em um mesmo macro ambiente. No entanto, o comportamento da variação do mesmo é semelhante em todos os modelos.

Observa-se que os modelos oriundos do GS medido na estação de Rio Grande apresentam probabilidade de falha superior aos modelos de Santa Pola, indicando que em termos médios o GS de Rio Grande é superior ao de Santa Pola. Esta situação se evidencia ao compararmos o modelo GS 1 a $28^{*}$, que utiliza a média dos CPs 1 a 28 expostos no Brasil, com o modelo GS (30, 31, 35 e 36), que utiliza a média dos testemunhos expostos na Espanha.

\section{CONCLUSÕES}

Após a realização da análise das variáveis meteorológicas mais influentes sobre o GS do concreto e a respectiva geração das equações para obtenção do GS, aplicou-se os resultados obtidos nos modelos de confiabilidade propostos chegando-se as seguintes conclusões:

- Os resultados obtidos demonstram que para a maioria dos casos analisados há uma boa correlação do GS com as variáveis meteorológicas estudadas, sendo possível obter boas estimativas do grau de saturação em função destas variáveis. Os modelos de geração de GS que apresentaram os melhores resultados foram os obtidos através da regressão linear múltipla, utilizando-se médias sazonais das variáveis. As equações obtidas fornecem um estimador aceitável do GS permitindo seu uso nos modelos de vida útil no que tange a penetração de íons cloreto.

- Os modelos apresentados destacaram o comportamento local do GS tanto para macro ambientes distintos (Rio Grande e Santa Pola) quanto dentro de um mesmo microambiente (Rio Grande) quando se comparam diferentes medições de GS em diferentes locais e condições de exposição. Observou-se a diferença em termos médios do GS medido em Santa Pola e em Rio Grande, fato possivelmente explicado pela diferença das características climáticas de cada região. Constatou-se também uma diferença considerável do GS medido na estação de medição a 1,2 km da costa em Rio Grande quando comparado ao medido por GUIMARÃES [37] junto à costa, apesar de ambas medições terem sido realizadas em zona de névoa marinha o GS próximo da costa foi aproximadamente $25,49 \%$ maior, constatando a influência da distância da linha d'água no GS, reforçando os resultados encontrados por MEIRA [38].

- Devido a inexistência de um estudo semelhante ao de GUIMARÃES [35] e GUIMARÃES [37] em estruturas situadas na Espanha e em ambiente similar ao desta pesquisa, não foi possível comparar os resultados obtidos dos modelos com dados de penetração de cloretos medidos em campo, porém, como os dados de GS foram obtidos de medições reais em ambos os locais os modelos podem ser validados em estudos futuros nos ambientes desta pesquisa.

- Os modelos de GS obtidos nesta pesquisa podem ser utilizados para estimar esta propriedade do concreto, porém, se faz necessário avaliar com muita atenção as características da estrutura estudada e sua similaridade com as condições dos testemunhos desta pesquisa, a observação de fatores como traço do concreto e o ambiente de exposição da estrutura devem ser levados em conta na escolha do modelo. Não havendo totais condições de semelhança entre a estrutura analisada e os testemunhos deste trabalho podem ser utilizados os modelos de GS obtidos para as médias dos CPs, considerando o ambiente de exposição mais semelhante. Cabe sempre ressaltar que os modelos obtidos foram gerados a partir de um conjunto de dados limitado e especifico às características apresentadas, logo sua utilização requer atenção.

Através da análise de confiabilidade apresentada pode-se destacar a relevância do uso do GS na avaliação da vida útil de estruturas de concreto armado sujeitas ao ataque de cloretos. A consideração da condição de concreto saturado leva a elevadas probabilidades de falha do elemento estrutural, o que na maioria das vezes não retrata a realidade, haja vista que dificilmente há saturação do concreto em microambiente de névoa. Os modelos gerados e utilizados no trabalho demonstram a sensibilidade do uso desta variável na estimativa de vida útil de elementos estruturais de concreto armado, porém, a escolha de qual modelo melhor se 
ajusta a realidade deve ser comprovada através de pesquisa experimental in loco para validação do mesmo.

\section{AGRADECIMENTOS}

O presente trabalho foi realizado com apoio da Coordenação de Aperfeiçoamento de Pessoal de Nível Superior - Brasil (CAPES) - Código de Financiamento 001.

Aos professores Miguel Ángel Climent e Guillem De Vera da Universidade de Alicante por coletar e ceder os dados de medição do GS da estação de Santa Pola.

Ao Centro de Investigación del Mar (CIMAR) da Universidade de Alicante, Espanha, por permitir a instalação da estação de exposição de testemunhos de concreto em ambiente marinho.

Ao CNPq pela bolsa de produtividade em pesquisa do $5^{\circ}$ autor.

\section{BIBLIOGRAFIA}

[1] BEUSHAUSEN, H., TORRENT, R., ALEXANDER, M.G. "Performance-based approaches for concrete durability: State of the art and future research needs". Cement and Concrete Research, v. 119, pp. 11-20, 2019.

[2] WALLY, G.B., MAGALHÃES, F.C., SILVA FILHO, L.C.P., "Conceitos e aspectos da abordagem com base no desempenho na avaliação da durabilidade de estruturas de concreto armado expostas a ambientes agressivos", Rev. Concreto \& Construções, ed. 100, pp. 81-85, Dec., 2020.

[3] SILVA, C.A., GUIMARÃES, A.T.C. "Avaliação de modelo de difusão considerando a variação no tempo do teor de cloretos na superfície do concreto". Revista Matéria, v.19, pp. 81-93, 2014.

[4] SHI, X., XIE, N., FORTUNE, K., et al., "Durability of steel reinforced concrete in chloride environments: An overview. Construction and Building Materials, v.30, pp.125-138, 2012.

[5] ANDRADE, M.C., Manual para diagnóstico de obras deterioradas por corrosão de armaduras, Tradução: Antônio Carmona e Paulo Helene, São Paulo, Pini, 1992.

[6] TORRENT, R., et al. Prescriptive Durability Specifications. In: BEUSHAUSEN, H.; LUCO, L. F. (coord.). Performance-Based Specifications and Control of Concrete Durability. New York: Springer, 2016, pp. 19-49.

[7] HELENE, P.R.L., Contribuição ao estudo da corrosão em armaduras de concreto armado, Tese (Livre Docente), EPUSP, São Paulo, SP, Brasil, 1993.

[8] GJøRV, O.E., TAN, K., ZHANG, M.. "Diffusivity of chlorides from seawater intohigh-strength ligh weight concrete." ACI Materials Journal, pp.447-452, set./out.1994.

[9] APOSTOLOPOULOS, C.A. PAPADAKIS, V.G. "Consequences of steel corrosion on the ductility properties of reinforcement bar", Construction and Building Materials, v. 22, Issue 12, pp. 2316-2324, Dec. 2008.

[10] HACKL, J., KOHLER, J. "Reliability assessment of deteriorating reinforced concrete structures by representing the coupled effect of corrosion initiation and progression by Bayesian networks", Structural Safety, v.. 62, pp. 12-23, 2016.

[11] VERA, G., ANTÓN, C., LÓPEZ, M.P., et al., "Depassivation time estimation in reinforced concrete structures exposed to chloride ingress: A probabilistic approach", Cement and concrete Composities, v. 79, p. 21-33, 2017.

[12] TUUTTI, K. "Service life of structures with regard to corrosion of embedded steel", In: PERFORMANCE OF CONCRETE IN MARINE ENVIRONMENT, ACI SP-65, 1980.

[13] YU, B., NING, C., LI B. "Probabilistic durability assessment of concrete structures in marine environments: Reliability and sensitivity analysis", China Ocean Engineering, v.31, pp.63-73, March 2017.

[14] GUIMARÃES, A.T.C., HELENE, P.R.L. "Modelos para Previsão de Vida Útil em Ambiente Marítimo." In: 42 ${ }^{\circ}$ Congresso Brasileiro do Concreto, 13-18, Fortaleza, CE: IBRACON, 2000.

[15] CLIMENT, M.A., VERA, G., LÓPEZ, J.F., et al. "A Test Method for Measuring Chloride Diffusion Coefficients Through Nonsaturated Concrete Part I: The Instantaneous Plane Source Diffusion Case”, Cement and concrete Research, v 32, pp. 1113-1123, 2002.

[16] NIELSEN, E.P., GEIKER, M.R., "Chloride diffusion in partially satured cementitions material”, Cement and Concrete Research, v. 33, pp. 133-138, 2003. 
[17] GUIMARÃES, A.T.C. "Transporte de íons cloreto no concreto: influência do grau de saturação. ”, In: PATORREB: $3^{\circ}$ Encontro sobre Patologia e reabilitação de edifícios $/ 3^{\circ}$ Congresso de Patologia y Rehabilitación de Edifícios, 27-32, Porto, 2009.

[18] MARTYS, N.S., "Diffusion in partially-saturated porous materials". Materials and Structures, v. 32, pp. 555-562, October, 1999.

[19] MERCADO-MENDOZA, H., LORENTE, S., BOURBON, X., "The Diffusion Coefficient of Ionic Species Through Unsaturated Materials", Transport in Porous Media, v. 96, Issue 3, pp. 469-481, Nov. 2012.

[20] MERCADO-MENDOZA, H., LORENTE, S., BOURBON, X., "Ionic aqueous diffusion through unsat rated cementitious materials - A comparative study", Construction and Building Materials, v. 51, pp. 1-8, 2014.

[21] PERAÇA, M.G.T., Modelos para estimativa do grau de saturação do concreto mediante variáveis ambientais que influenciam na sua variação, Tese de M.Sc., FURG, Rio Grande, RS, Brasil, 2009.

[22] VÍLCHEZ, E.F., Estudio de la influencia de las varibles meterologicas em el grado de saturacion em hormigones expuesto a un ambiente marino atmosferico mediterraneo, Tese de M.Sc., UA, Alacant, Alicante, Espanha, 2011.

[23] HALDAR, A., MAHADEVAN, S., Probability, Reliability, and Statistical Methods in Engineering Design, EUA, John Wiley \& Sons, 2000.

[24] ANDRADE, J.J.O., Contribuição à previsão da vida útil das estruturas de concreto armado atacadas pela corrosão de armaduras: iniciação por cloretos, Tese de D.Sc., UFRGS, Porto Alegre, RS, Brasil, 2001.

[25] MAGALHÃES, F.C., Proposição de um modelo para penetração e íons cloreto em concreto: estudos dos parâmetros intervenientes e análise probabilística, Tese de D.Sc., UFRGS, Porto Alegre, RS, Brasil, 2018.

[26] MELCHERS, R.E., BECK, A.T. Structural Realiability Analysis and Prediction, EUA, Wiley, 2018.

[27] SANTIAGO, W.C. Estudo da Não Conformidade de Concretos Produzidos no Brasil e sua Influência na Confiabilidade Estrutural, Tese de M.Sc., USP, São Carlos, SP, Brasil, 2011.

[28] MAGALHÃES, F.C.; REAL, M. V.; SILVA FILHO, L.C.P. "The problem of non-compliant concrete and its influence on the reliability of reinforced concrete columns" Materials and Structures, v. 49, pp. 14851497, 2016.

[29] ANG, A.H-S., TANG, W.H. Probability concepts in engineering planning and design. Volume II: Decision, risk, and reliability. New York, John Wiley \& Sons, 1984.

[30] BENTZ, E.C., "Probabilistic modeling of service life for structures subjected to chlorides", ACI Materials Journal, v. 100, Issue 5, pp. 391-397, 2003.

[31] GJØRV, O.E. Projeto da durabilidade de estruturas de concreto em ambientes de severa agressividade, Trad. Leda Maria Marques Dias Beck, São Paulo, Oficina de Textos, 2015.

[32] FAVRETTO, F., Modelos para estimativa do grau de saturação do concreto em função das condições meteorológicas de dois macro ambientes distintos e sua contribuição na estimativa de vida útil de projeto, Tese de M.Sc., FURG, Rio Grande, RS, Brasil, 2019.

[33] DEVORE, J.L. Probabilidade e Estatística para Engenharia e Ciências, São Paulo, Pioneira Thomson, 2006.

[34] PETERNELLI, L.A., NOTAS DE AULA, www.16032004/materiais/CAPITULO9.pdf, Acessado em maio de 2018.

[35] GUIMARÃES, A.T.C., Vida útil de estruturas de concreto armado em ambientes marítimos, Tese de D.Sc., Universidade de São Paulo - PCC/USP, Engenharia Civil, São Paulo SP, Brasil, 2000.

[36] BRETANHA, S.F., Variação do Grau de Saturação do Concreto em Ambiente Marítimo, Tese de M.Sc., FURG, Rio Grande, RS, Brasil, 2004.

[37] GUIMARÃES, A.T.C., Grau de Saturação: Sua Variação com o Tipo de Concreto e sua Influência na Difusão de ions Cloreto, Tese de Pós D.Sc, EPUSP, São Paulo, SP, Brasil, 2005.

[38] MEIRA, G.R., Agressividade por Cloretos em Zona de Atmosfera Marinha Frente ao Problema da Corrosão em Estruturas de Concreto Armado, Tese de D.Sc, UFSC, Florianópolis, SC, Brasil, 2004.

[39] RODRIGUES, F.T., Medição do grau de saturação e a difusão de íons cloreto em concretos executados com cimento de alta resistência inicial, Tese de M.Sc., FURG, Rio Grande, RS, Brasil, 2009. 


\section{ORCID}

Felipe Favretto

https://orcid.org/0000-0001-7788-8739

Fabio Costa Magalhães

https://orcid.org/0000-0001-8463-1501

André Tavares da Cunha Guimarães

https://orcid.org/0000-0002-3229-9911

Miguel Ángel Climent

https://orcid.org/0000-0001-9026-930X

Mauro de Vasconcellos Real

https://orcid.org/0000-0003-4916-9133 